\title{
HOSPITAIS UNIVERSITÁRIOS: PASSADO, PRESENTE E FUTURO
}

A.C. MedicI

TrabalhorealizadonoBancolnteramericanode Desenvolvimento, Washington,D.C.

RESUMO - OBjetivo. 0 presente artigo discute a evolução dos hospitais universitários, suas características, funcionamento e financiamento, e seu papel no ensino e pesquisa médicos.

Métodos. Baseia-se nas informações e conclusões de um seminário organizado pela Organização Mundial da Saúde sobre os hospitais universitários de 22 países.

Resultados. $\mathbf{O}$ artigo avalia que essas instituições estão crescentemente inadaptadas à realidade do setor saúde em que se inserem e aos desafios impostos pelas mudanças profundas atualmente em curso no sistema de saúde da maioria dos países.

Conclusão. Conclui-se pela necessidade de reforma dos hospitais universitários, e algumas estratégias para essas reformas são apresentadas e discutidas.

UnIteRmos: Hospitais Universitários. Educação Médica.

\section{INTRODUÇÃO}

Hospitais de ensino são tão antigos como o conceito de saúde que surgiu com o renascimento. No entanto, a medicina flexeneriana e seu impacto no aumento da especialização, a partir do início do século $X X$, ampliou o escopo destas instituições, tornando obrigatório seu vínculo orgânico e dependência institucional junto as Faculdades de Medicina.

Ainda que mantidos, em muitos casos, por verbas públicas, os hospitais universitários cresceram nos últimos setenta anos, em sua grande maioria, como instituições independentes, distantes do perfil epidemiológico das populações e dominadas pelos interesses dos médicos especialistas. Estes os utilizavam, seja como meios de experimentação de novas tecnologias médicas, seja como loci de recrutamento de novos médicos que iriam engrossar as fileiras das "guildas" de especialistas, aumentando o poder social e econômico daqueles que se encontravam no topo da pirâmide de

\footnotetext{
*Correspondência:

8207 Lilly Stone Drive-Bethesda (MD)-20817-USA E-mail:andrem@iadb.org
}

cada especialidade ou sub-especialidade médica.

Porém, a partir dos anos setenta, novos desafios começam a definir mudanças no horizonte dos serviços de saúde, onde cabe destacar: a) a atenção primária como prática associada ao conceito de democratização da saúde; b) as técnicas de prevenção que ganham força como instrumento para prolongar a vida e reduzir custos dos sistemas de saúde; c) o crescimento da atenção médica baseada no conceito de seguro, mudando as práticas das instituições que administram os planos de saúde e submetendo a atenção médica a rotinas, procedimentos e práticas mais padronizadas, bem como a controles externos, com a perspectiva de obter resultados mais custo-efetivos; d) o aumento do conhecimento e da regulação do setor, estabelecendo-se um vínculo entre saúde, ambiente, trabalho, alimentação e transporte; e) a multidisciplinariedade crescente da atenção a saúde; f) o crescimento dos mecanismos de defesa do consumidor e do aparato judicial contra práticas que antes eram inquestionáveis, por serem monopólio do saber da profissão médica, a qual nunca era posta em cheque.

Por outro lado, as necessidades de ra- cionalizar gastos com saúde têm fragilizado os hospitais de ensino, que costumam ser muito dispendiosos, dado não apresentarem uma relação eficiente entre custos e resultados. Estes são apenas alguns dos elementos que "quebraram as vidraças" dos hospitais universitários e trouxeram o debate acerca da formação em saúde para as ruas.

Mas como as instituições de ensino em saúde têm recebido estas mudanças ao nível mundial? Como médicos e professores universitários têm reagido aos novos conceitos e proposições? A realidade é que o debate apenas está começando, inclusive nos países desenvolvidos. Com base nestas preocupações, a Organização Mundial da Saúde realizou em 1995 um seminário internacional buscando avaliar o "estado da arte" dos hospitais universitários. Os resultados foram publicados em um estudo que faz uma avaliação destas instituiç̧ões em 22 países'.

\section{Hospital universitário: conceito e formas de operação}

A concepção tradicional define um hospital universitário $(\mathrm{HU})$ como uma instituição que se caracteriza: (a) por ser um pro- 


\begin{tabular}{|c|c|c|c|c|c|c|}
\hline \multirow[t]{2}{*}{ País } & \multirow[t]{2}{*}{$\begin{array}{c}\text { População } \\
\text { (milhões) } \\
\text { I994 }\end{array}$} & \multirow[t]{2}{*}{$\begin{array}{c}\text { PIB } \\
\text { Percapita } \\
\text { (US\$) 1994 }\end{array}$} & \multirow{2}{*}{$\begin{array}{c}\text { Esperança } \\
\text { de Vida } \\
\text { (Anos) I994 } \\
\text { ALTA RENDA }\end{array}$} & \multirow[t]{2}{*}{$\begin{array}{l}\text { Índice de } \\
\text { Desenvol. } \\
\text { Humano }\end{array}$} & \multirow[t]{2}{*}{$\begin{array}{c}\text { Taxa de } \\
\text { Mortalidade } \\
\text { Infantil }(0 / 00)\end{array}$} & \multirow[t]{2}{*}{$\begin{array}{c}\text { Taxa de } \\
\text { Urbanização } \\
(\%)\end{array}$} \\
\hline & & & & & & \\
\hline Suiça & 7 & 37930 & 78 & 0,978 & 6,8 & 60 \\
\hline Japão & 125 & 34630 & 79 & 0,983 & 4,8 & 77 \\
\hline Suécia & 9 & 23530 & 78 & 0,977 & 5,8 & 84 \\
\hline França & 58 & 23420 & 78 & 0,971 & 7,7 & 74 \\
\hline Holanda & 15 & 22010 & 78 & 0,970 & 6,8 & 89 \\
\hline Austrália & 18 & 18000 & 77 & 0,972 & 8,7 & 85 \\
\hline Reino Unido & 58 & 18340 & 76 & 0,964 & 7,0 & 89 \\
\hline \multicolumn{7}{|c|}{ MÉDIA RENDA } \\
\hline Coréia do Sul & 44 & 8260 & 71 & 0,872 & 22,0 & 72 \\
\hline Chile & 14 & 3520 & 72 & 0,864 & 17,0 & 86 \\
\hline Jamaica & 3 & 1540 & 74 & 0,736 & 15,0 & 52 \\
\hline Colômbia & 36 & 1670 & 70 & 0,770 & 38,0 & 70 \\
\hline Marrocos & 26 & $1 \mid 40$ & 65 & 0,433 & 72,0 & 48 \\
\hline Filipinas & 67 & 950 & 65 & 0,603 & 42,0 & 43 \\
\hline Albânia & 3 & 380 & 73 & 0,699 & - & 35 \\
\hline \multicolumn{7}{|c|}{ BAIXA RENDA } \\
\hline Egito & 57 & 720 & 62 & 0,389 & 59,0 & 47 \\
\hline Indonésia & 190 & 880 & 63 & 0,515 & 68,0 & 31 \\
\hline Paquistão & 126 & 430 & 60 & 0,311 & $|0|, 0$ & 32 \\
\hline China & || $9 \mid$ & 530 & 69 & 0,566 & 29,0 & 33 \\
\hline Benin & 5 & 370 & 50 & 0,113 & 88,0 & 38 \\
\hline Nigéria & 108 & 280 & 52 & 0,246 & 99,0 & 35 \\
\hline Tanzânia & 29 & 140 & 51 & 0,270 & 104,0 & 33 \\
\hline Vietnã & 72 & 200 & 68 & 0,472 & 39,0 & 22 \\
\hline
\end{tabular}

longamento de um estabelecimento de ensino em saúde (de uma faculdade de medicina, por exemplo); (b) por prover treinamento universitário na área de saúde; (c) por ser reconhecido oficialmente como hospital de ensino, estando submetido à supervisão das autoridades competentes; (d) por propiciar atendimento médico de maior complexidade (nível terciário) a uma parcela da população. Mas nas últimas duas décadas, ampliou-se nos países desenvolvidos a autonomia dos hospitais universitários, os quais passam a manter funções definidas nos sistemas de saúde e a se subordinar progressivamente à lógica desses sistemas.
Do ponto de vista prático, a realidade dos 22 países analisados mostra que um hospital universitário é entendido, antes de tudo, como um centro de atenção médica de alta complexidade que: (a) tem importante papel no atendimento médico de nível terciário; (b) apresenta forte envolvimento em atividades de ensino e pesquisa relacionada ao tipo de atendimento médico que dispensa; (c) atrai alta concentração de recursos físicos, humanos e financeiros em saúde e; (d) exerce um papel político importante na comunidade que está inserido, dada sua escala, dimensionamento e custos.

As tabelas 1, 2 e 3 em anexo mostram algumas das características dos países anali- sados no que se refere a seus indicadores econômicos e sociais (países de alto, médio e baixo índice de desenvolvimento) e relacionados aos sistemas de saúde, assim como os dados relativos aos hospitais universitários. Neste último campo, as informações são bastante incompletas, sobretudo para os países de médio e baixo grau de desenvolvimento. Ainda que a importância dos hospitais universitários tenha alguma relação com o nível de desenvolvimento, algumas características destes estabelecimentos são mais ou menos gerais. Os HU representam uma parcela importante do gasto total com saúde, que pode variar de 4,5\% (Colômbia) até 21\% (Coréia). A mé- 


\section{Tabela 2- Indicadores do sistema de saúde selecionados}

\begin{tabular}{|c|c|c|c|c|c|c|}
\hline \multirow[t]{2}{*}{ País } & $\begin{array}{l}\text { Empregos de } \\
\text { Saúde por } 1000 \\
\text { habitantes }\end{array}$ & $\begin{array}{l}\text { Médicos por } \\
1000 \\
\text { habitantes }\end{array}$ & $\begin{array}{c}\text { Percentagem } \\
\text { de Médicos } \\
\text { Especialistas }\end{array}$ & $\begin{array}{c}\text { Leitos } \\
\text { Hospitalares } \\
\text { por } 1000 \\
\text { habitantes }\end{array}$ & $\begin{array}{c}\text { Gasto em } \\
\text { Saúde } \\
\text { (\% PIB) }\end{array}$ & $\begin{array}{c}\text { Gastos } \\
\text { Hospitalares } \\
\text { (\% gasto em } \\
\text { Saúde) }\end{array}$ \\
\hline & \multicolumn{6}{|c|}{ ALTA RENDA } \\
\hline Suiça & - & 3,0 & 70 & 10,2 & 7,5 & 43 \\
\hline Japã̃o & 16,7 & 1,7 & 43 & 13,6 & 6,5 & 60 \\
\hline Suécia & 40,7 & 3,0 & 67 & 6,2 & 8,8 & 55 \\
\hline França & 23,8 & 2,7 & 48 & 9,7 & 8,9 & 57 \\
\hline Holanda & 23,7 & 2,2 & 57 & 5,9 & 7,9 & 55 \\
\hline Austrália & 16,8 & 2,2 & 34 & 5,6 & 7,7 & 50 \\
\hline Reino Unido & 17,6 & 2,0 & 49 & 4,8 & 6,1 & 51 \\
\hline \multicolumn{7}{|c|}{ MÉDIA RENDA } \\
\hline Coréia do Sul & 10,0 & 1,1 & 55 & 3,0 & 6,6 & 52 \\
\hline Chile & 6,5 & 1,0 & & 3,2 & 4,7 & 48 \\
\hline Jamaica & - & 0,5 & - & 0,0 & - & - \\
\hline Colômbia & 3,6 & 1,0 & 41 & 1,3 & 4,0 & - \\
\hline Marrocos & 1,3 & 0,3 & 49 & 1,2 & 2,6 & - \\
\hline Filipinas & 10,3 & 1,1 & - & 1,4 & 2,0 & - \\
\hline Albânia & & 1,8 & 25 & 3,0 & 4,0 & - \\
\hline \multicolumn{7}{|c|}{ BAIXA RENDA } \\
\hline Egito & - & 1,3 & - & 2,0 & 2,6 & - \\
\hline Indonésia & 1,6 & 0,1 & 33 & 0,6 & 2,0 & - \\
\hline Paquistão & & 0,4 & - & 0,6 & 3,4 & - \\
\hline China & 4,4 & 1,5 & - & 2,4 & 3,5 & - \\
\hline Benin & 0,7 & 0,1 & 37 & 0,9 & 4,3 & 58 \\
\hline Nigéria & & 0,2 & - & 1,4 & 2,7 & \\
\hline Tanzânia & 0,9 & 0,0 & 27 & 1,1 & 4,7 & 55 \\
\hline Vietnã & - & 1,1 & - & 3,3 & 2,1 & . \\
\hline
\end{tabular}

dia dos países europeus onde foram levantadas as informações se situa entre $7 \%$ e 12\%. Os leitos dos HU representam entre $5 \%$ (lapão) e $21 \%$ (Coréia) do total de leitos do país e a participação no total de gastos hospitalares pode variar de 9\% (Colômbia) a 40\% (Coréia). Assim, ao utilizarem alta tecnologia e envolverem ensino e pesquisa, seu peso na despesa com saúde é o dobro de sua participação no volume de atendimento. São, portanto, hospitais caros. Como porcentagem do PIB, os gastos dos hospitais universitários podem variar de 0,1\% (Marrocos) a I,4\% do PIB (Coréia).

Os HU respondem por uma atenção médica basicamente curativa, havendo pouca preocupação com a prevenção. No caso dos países desenvolvidos tal fato começa lentamente a mudar, especialmente no que se refere ao gerenciamento de enfermidades crônicas, como a diabetes e as enfermidades cardiovasculares, onde a educação do paciente e da família é condição sine-qua-non para a eficácia dos tratamentos.

Há um certo consenso de que os $\mathrm{HU}$ deveriam mudar suas estratégias e se integrarem mais com as comunidades onde se baseiam, definindo novas formas de tratamento e visitas domiciliares, aumentando o atendimento ambulatorial, etc. A vantagem desta integração estaria no fato de que, como instituiç̧ões preocupadas com o ensino e a pesquisa, os HU poderiam experimentar, de forma pioneira, novas formas de micro-gerenciamento da saúde, as quais poderiam ser transferidas para os demais hospitais, uma vez testadas.

Ainda que alguns $\mathrm{HU}$ prestem serviços de atenção primária, sobretudo nos países de menor desenvolvimento, os participantes do encontro promovido pela OMS foram muito claros em defender a posição de que a função básica do HU é a prestação de serviços de alta complexidade e tecnologia. 
Tabela 3 - Indicadores dos Hospitais Universitários (HU) selecionados

\begin{tabular}{|c|c|c|c|c|c|c|}
\hline País & $\begin{array}{c}\text { Leitos de } \\
\text { HU/Total } \\
\text { de Leitos (\%) }\end{array}$ & $\begin{array}{c}\text { Gasto dos } \\
\text { HU/Gasto } \\
\text { Total e/ } \\
\text { Saúde (\%) }\end{array}$ & $\begin{array}{c}\text { Gasto dos } \\
\text { HU/Gasto } \\
\text { Hospitalar } \\
\text { (\%) }\end{array}$ & $\begin{array}{l}\text { Duração do } \\
\text { Curso de } \\
\text { Medicina } \\
\text { (anos) }\end{array}$ & $\begin{array}{l}\text { Duração dos } \\
\text { Cursos de } \\
\text { Especializ. } \\
\text { (anos) }\end{array}$ & $\begin{array}{c}\text { Gasto com } \\
\text { Hospitais } \\
\text { Universitários } \\
\text { (\% PIB) }\end{array}$ \\
\hline \multicolumn{7}{|c|}{ ALTA RENDA } \\
\hline Suíça & II & 8,3 & 19,2 & 7 & 5 & 0,6 \\
\hline Japão & 5 & 6,6 & 11 & - & - & 0,4 \\
\hline Suécia & 19 & 11,7 & 21,2 & 6 & 7 & 1,0 \\
\hline França & 17 & 11,7 & 24,8 & 6 & 5 & 1,0 \\
\hline Holanda & 8 & 7,7 & 14,0 & 6 & 5 & 0,6 \\
\hline Austrália & - & - & - & 6 & 5 & - \\
\hline Reino Unido & - & - & - & 5 & 7 & - \\
\hline \multicolumn{7}{|c|}{ MÉDIA RENDA } \\
\hline Coréia do Sul & 21 & 20,8 & 40 & 6 & 5 & 1,4 \\
\hline Chile & - & - & - & 7 & - & - \\
\hline Jamaica & - & - & - & - & - & - \\
\hline Colômbia & 10 & 4,5 & 9,3 & 6 & 4 & 0,2 \\
\hline Marrocos & 17 & 4,9 & - & 7 & - & 0,1 \\
\hline Filipinas & - & - & - & 5 & - & - \\
\hline Albânia & 20 & - & - & 6 & 5 & - \\
\hline \multicolumn{7}{|c|}{ BAIXA RENDA } \\
\hline Egito & 18 & - & - & - & - & - \\
\hline Indonésia & - & - & - & 7 & 4 & - \\
\hline Paquistão & - & - & - & 5 & 2 & - \\
\hline China & - & - & - & - & - & - \\
\hline Benin & 14 & 8,1 & 13,9 & - & - & 0,3 \\
\hline Nigéria & - & - & - & - & - & - \\
\hline Tanzânia & 5 & 12,2 & 22,2 & 5 & 3 & 0,6 \\
\hline Vietnã & - & - & - & - & - & - \\
\hline
\end{tabular}

$\mathrm{O}$ argumento é basicamente de natureza econômica, isto é, seria um desperdício de recursos utilizar estruturas pensadas para oferecer atividades de alta tecnologia como prestadoras de serviços básicos.

A posição atualmente defendida pelos gerentes dos $\mathrm{HU}$ e diretores das Faculdades de Medicina, no entanto, não equaciona questões fundamentais como a formação prática de médicos e equipes de saúde que vão trabalhar em atenção primária e prevenção, assim como sua relação com o hospital. Algumas soluções práticas existem para o problema:

a) utilizar outros estabelecimentos de saúde (hospitais de menor complexidade, redes de atenção primária, estruturas de saúde de família, etc.) como locais de ensino alternativos para estas modalidades, de forma integrada aos planos curriculares das faculdades de medicina, ou;

b) reformular o conceito de ensino em saúde sem vinculá-lo necessariamente a existência de hospitais universitários. Neste último caso haveria o abandono da idéia de $\mathrm{HU}$, ainda que pudessem ser contratados hospitais terciários (com estrutura de pesquisa) para o ensino de ações que envolvessem procedimentos de alta tecnologia. Alguns países, como Chile, Colômbia, Austrá- lia e Paquistão já vem experimentando o ensino de atividades de atenção primária fora dos HU.

Dada a tendência internacional à crescente des(h)ospitalização da saúde, os HU passam a ter que se adaptar a novas formas de cuidado como a internação domiciliar, postos avançados em pequenas cidades, acomodações especiais para familiares, no caso de hospitais pediátricos, uso de estratégias de ajuda voluntária em hospitais, etc. Poucos países, no entanto, vêm experimentando inovações neste campo, a partir dos HU. As experiências de vanguarda são mais fáceis de encontrar, seja nas institui- 
ções de seguro saúde e suas redes de atenção próprias ou contratadas, seja no setor público de base local.

O fato de dispor de pessoal mais qualificado ou mais recursos físicos e equipamentos não leva necessariamente os $\mathrm{HU}$ a prestar uma atenção médica de melhor qualidade. A experiência tem demonstrado que os HU, a despeito de terem mais recursos físicos e humanos, apresentam, em muitos casos, forte deterioração de seus padrões de atendimento.

Tal situação é particularmente grave, porque o não cumprimento de padrões de qualidade e de conduta médica adequada podem deformar o profissional em treinamento por toda a sua vida. Há um consenso, neste caso, de que os HU deveriam ser instituições modelares, constantemente avaliadas e acreditadas por instituições externas ao hospital. Muitos dirigentes dizem que isto não ocorre dada a inexistência de financiamento e incentivos adequados. No entanto, o mau gerenciamento dos $\mathrm{HU}$ e a relativa estabilidade e impunidade de seus dirigentes têm sido, em todo o mundo, um dos principais fatores responsáveis por esta deterioração.

Se considerado válido o argumento de que os $\mathrm{HU}$ devem ser somente unidades de terceiro nível de atenção, o acesso a estes hospitais deveria ter como "gate keeper" uma rede de referência, onde as unidades de menor complexidade resolveriam a maior parte dos problemas hoje tratados por estes hospitais. Na prática, no entanto, não é isso que acontece. Como os HU estão pouco integrados aos demais níveis de atenção e têm, em geral, total autonomia gerencial em relação aos sistemas de saúde, acabam atendendo a todos os níveis de atenção, com um alto risco de encaminhar os paciente para níveis de complexidade assistencial maiores e mais caros que os necessários para resolver os problemas relacionados ao diagnóstico que apresentam. Na verdade, os hospitais universitários são mais caros por isto e não simplesmente por incorporarem atividades docentes-assistenciais, como se diz no senso comum.

$O$ fato de existir um sentimento comum entre a população de que "quanto maior e mais complexo o estabelecimento, melhor o atendimento", perpetua a mística de que os hospitais universitários resolveriam de forma mais adequada todo problema de saúde que se apresente. Os dados não necessariamente confirmam essa crença popular, a qual é fruto da assimetria de informação característica do setor saúde.

\section{O ensino e a pesquisa nos hospitais universitários}

A análise do ensino nos HU envolve uma série de aspectos, dentre os quais cabe destacar: a) deveriam ser eles as únicas instituições práticas de formação em saúde?; b) qual o tipo de treinamento que os $\mathrm{HU}$ deveriam prover? c) quantos médicos deveriam ser formados? d) que tipo de treinamento deveria ser dado a outros profissionais? e) como financiar as atividades de Ensino no interior dos HU?

Ainda que os $\mathrm{HU}$ sejam as principais instituições que complementam, em termos práticos, o ensino acadêmico nas profissões de saúde, vem crescendo rapidamente o número de hospitais e outros estabelecimentos não-universitários que exercem estas funções. A realidade mostra que HU não são imprescindíveis. Ao contrário, são cada vez mais dispensáveis, quando se pensa que as atividades de saúde passam crescentemente pela interdisciplinariedade das profissões e campos de conhecimento e pela mudança radical nas formas de atenção médica, as quais se distanciam crescentemente do conceito tradicional de hospital. A prática do setor saúde é muito mais rica e diversificada que o mundo dos HU. Portanto, ainda que deva existir uma interação entre ensino e prática em saúde, é mais adequado conduzir o ensino em saúde pela prática do que proceder o caminho inverso, que é o traçado pelos $\mathrm{HU}$.

A questão de quantos profissionais se deve formar deveria ter um paralelo com as necessidades de saúde. Mas isso não ocorre necessariamente. Mesmo os HU públicos respaldam-se na abstrata (e muitas vezes anti-social) figura da autonomia universitária para oferecer cursos e vagas em profissões de saúde totalmente descoladas das necessidades, como ocorre no Uruguai, Argentina e algumas partes do Brasil, especialmente quando o financiamento da educação não provém do mercado, mas sim de fontes orçamentárias públicas, não relacionadas com a demanda pela profissão.

Determinar quantos médicos ou profissionais são necessários, no entanto, não é um processo trivial. A quantidade de variáveis necessárias e a subjetividade das análises envolvidas pode estabelecer um sistema de equações com múltiplas hipóteses e resultados. O mesmo diz respeito a qual a proporção adequada entre médicos generalistas e especialistas a se formar. Ao longo do seminário realizado pela OMS se estabeleceu que seria uma aproximação razoável a proporção de 50\% para cada. Neste particular, países como Japão, França, Austrália e Inglaterra teriam menos de $50 \%$ de especialistas enquanto que outros como Suíça, Suécia e Holanda teriam mais do que essa proporção. De qualquer forma, situações absurdas são as encontradas no Uruguai e Argentina, com proporções superiores a $80 \%$.

Quanto ao financiamento dos $\mathrm{HU}$, a maioria deles está sob a administração de faculdades de medicina ou universidades, isto é, depende do orçamento público para a sua manutenção. Com isso, planejam suas atividades de forma independente da demanda e do mercado, praticando em larga escala o que é ditado pelas necessidades do corpo docente.

Nos últimos anos têm crescido o núme- 
ro de HU que passa a ter parte do seu financiamento atrelado aos serviços que são prestados para a comunidade. Tal fato, em alguns países, tem gerado uma série de conflitos administrativos entre Ministérios da Educação e Saúde, a respeito de quais deveriam ser as reais funções destes estabelecimentos e a que tipo de lógica deveriam responder: a do ensino ou a dos serviços.

Os resultados do seminário da OMS mostram que o tipo de pesquisa que se desenvolve nos HU é a pesquisa clínica, através da observação dos pacientes. No entanto, nos países em desenvolvimento, a pesquisa não tem sido considerada uma atividade essencial dentre as tarefas dos HU. Mesmo nos países desenvolvidos, boa parte da pesquisa que se desenvolvia nas décadas de sessenta e setenta nos HU é hoje canalizada através dos Institutos de Pesquisa e das indústrias farmacêutica e de equipamentos médicos. Os altos custos do desenvolvimento científico e tecnológico e os limites dos orçamentos públicos para a pesquisa fazem com que os $\mathrm{HU}$ tenham cada vez menos capacidade institucional para se envolverem em processos complexos de pesquisa básica e aplicada em saúde.

A tendência é que os hospitais universitários possam participar como parceiros em experimentos científicos, auxiliando os Institutos de Pesquisa ou sendo financiados parcialmente por indústrias. Mas cada vez mais estão longe de se envolver isoladamente em linhas de desenvolvimento científico e tecnológico.

\section{Aspectos institucionais e sociais dos hospitais universitários}

A maioria dos países participantes não considera que os $\mathrm{HU}$ devam ser orientados para a solução de problemas sociais. Eles não são instituições filantrópicas nem se orientam diretamente para cumprir atividades voltadas a mitigar a pobreza e melhorar a distribuição de renda. Mesmo assim, eles podem oferecer serviços gratuitos a populações carentes que não poderiam, regularmente, pagar por serviços de saúde de alta complexidade.

Como foi visto anteriormente, os $\mathrm{HU}$ são instituições caras, por concentrarem atendimentos de alta complexidade, além de atividades que mesclam atos médicos com procedimentos didáticos. Os estudos realizados na Austrália mostram que, considerando-se o tratamento de um conjunto de enfermidades selecionadas, o custo dos hospitais universitários é I $2 \%$ mais elevado que o dos hospitais não-universitários de alta tecnologia. É possível que atividades que envolvam ensino incluam uma certa dose de ineficiência técnica implícita ao processo didático. No caso dos Hospitais da Coréia do Sul, a média de custos relativa aos HU é 28\% maior que a dos demais hospitais para um mesmo conjunto de enfermidades tratadas e procedimentos clínicos selecionados.

Por apresentarem custos mais elevados, dificilmente os hospitais universitários poderiam ser competitivos em sistemas de "fee-for-service" e seriam pouco atrativos aos planos de seguro médico. A tendência, portanto, é que se mantenham sendo custeados pelo setor público, com boa parte dos serviços prestados de forma gratuita ou subsidiada. Mesmo assim, deveriam envidar esforços para ter informação financeira relacionada aos custos, além de buscar fontes alternativas de financiamento para a atenção médica de forma separada das distintas funções adicionais (ensino e pesquisa) que os diferenciam dos demais hospitais.

Em outras palavras, os $\mathrm{HU}$ deveriam ser unidades de venda ou asseguramento de serviços de alta tecnologia em saúde para o Governo, os planos de saúde e as pessoas físicas e jurídicas, especialmente nas atividades onde funcionam como centro de referencia ou são monopolistas na oferta dos serviços. Esta deveria ser a maneira pela qual poderiam financiar em parte seus orçamentos, de forma complementar às funções de ensino e pesquisa, as quais poderiam ser remuneradas através de outras formas de relacionamento com o setor público ou privado. As próprias universidades ou faculdades de medicina deveriam ser encaradas como "clientes" dos HU e não como órgãos controladores ou gestores dos mesmos. Todos esses aspectos, no entanto, envolveriam a busca por uma maior eficiência e racionalidade na gestão destas instituições.

O estudo da OMS demonstrou que, embora a maioria dos $\mathrm{HU}$ tenham personalidade jurídica pública, o fato deles serem públicos ou privados não acarreta nenhuma diferença substantiva nos problemas que em geral apresentam. HU privados também têm sido subsidiados de forma pouco custo efetiva e aparentemente são tão ineficientes como os públicos. Tal fato se apresenta mais como uma questão de falta de incentivos e mecanismos adequados de financiamento e menos como um problema crônico de gerenciamento.

Muitos países (em especial os países desenvolvidos), ja começaram desde os anos oitenta a utilizar a definição de "standards" para acreditação, tecnologia, controle de qualidade e normas para referenciar pacientes aos HU. Mas nos países em desenvolvimento, os HU ainda não se integram a uma rede de serviços hierarquizada e continuam sujeitos a grande irracionalidade gerencial e assistencial. Tal fato tem implicações, inclusive sobre a eqüidade das ações promovidas pelos hospitais universitários, já que fatores como localização do hospital, facilidades de transporte e mecanismos de acesso a informação tem influenciado fortemente a natureza da demanda por estes hospitais. Por outro lado, o fato de oferecerem serviços gratuitos e manterem uma imagem positiva de resolutividade con- 
tinua atraindo as populações pobres para utilizá-los na solução de qualquer problema de saúde, sem que haja uma triagem prévia dos tipos de problemas a serem tratados nestes hospitais.

Neste sentido, as populações de menor renda poderiam ser melhor servidas, com menor custo, maior efetividade e maior eficiência alocativa dos recursos em unidades de menor complexidade, caso fosse possível fazer com que os $\mathrm{HU}$ se submetessem a uma adequada integração com a rede de serviços de forma hierarquizada.

Em geral, toda a nação se orgulha de ter um hospital universitário (ou se envergonha quando ele se encontra em franca deterioração). Um exemplo que comprova esta afirmação gira em torno do debate sobre a reforma do Hospital das Clínicas do Uruguai, ao longo dos anos noventa, onde a má qualidade da atenção médica prestada pelo referido Hospital tem gerado forte mobilização de corações e mentes em todo o país, sem que haja uma solução de consenso. Com taxas de infecção hospitalar superiores a 20\% em algumas alas e com baixíssimo nível de resolutividade em face dos quase 3.000 funcionários do hospital, ele tem sido considerado um mau exemplo por todos, embora a Universidade venda sempre a imagem de que o problema do hospital não é sua gestão ineficiente e sua inadequada inserção na rede de serviços, mas sim a falta de recursos. Como um estabelecimento encarado como de "tecnologia de ponta", falhas na qualidade de um hospital universitário podem ferir a auto-estima nacional associada a um dos aspectos mais vulneráveis do imaginário social de uma nação: sua inteligência.

Como último aspecto institucional relevante, vale a pena destacar que os hospitais universitários são fontes de prestígio e poder da classe médica e estão sujeitos ao forte controle dos grêmios de profissionais de saúde, os quais podem manipular sua gestão, em muitos casos, como aparelhos sindicais; seja pelo lado da classe dos "professores universitários", seja pela própria classe médica. Isto os transforma em instituições de difícil manejo e controle social, fazendo com que sejam vulneráveis ao veIho dilema da apropriação privada do espaço público por grupos de interesse, sem o controle social que muitas vezes poderia ser exercido através da regulação pública ou do mercado.

\section{O futuro dos hospitais universitários e sua integração com as redes assis- tenciais}

Apesar de todos os problemas colocados, é difícil considerar que os hospitais universitários estão em extinção e serão substituídos por instituições mais eficientes a curto e médio prazo. Como é natural, os médicos exercem forte poder gravitacional em todas as instituiçõ̃es de saúde, especialmente nos $\mathrm{HU}$ e dificilmente deixariam que estes escapassem dos interesses coletivos dos grêmios de especialistas médicos, de professores universitários ou simplesmente de funcionários do hospital. O grande debate nos dias de hoje é, portanto, dados os conflitos de interesses existentes, como tornar os HU instituições que, no marco de suas atribuições, possam se integrar à rede de saúde e contribuir para a eficiência e maior racionalidade no funcionamento desses sistemas.

Integrar os hospitais universitários em redes de referência e contra-referência pode ser muito difícil, particularmente quando se leva em conta os interesses das corporações médicas universitárias em manter a desvinculação entre as funções do hospital e as reais demandas por saúde, com base em argumentos científicos e acadêmicos, conservando seu poder sobre os rumos dos $\mathrm{HU}$. A tendência, nas regiões onde os HU são financiados pelo lado da oferta com recursos públicos, consiste em que estes hospitais criem suas próprias redes de cuidados primá- rios e secundários, duplicando esforços com a rede pública de saúde já existente.

Uma alternativa para solucionar este problema consiste em mudar a lógica de financiamento das atividades docentes em saúde, submetendo-as progressivamente a processos onde sejam remunerados pelo lado da demanda, de forma competitiva com outras instituições que poderiam cumprir atividades docentes. Trata-se neste caso de desenvolver redes docentes-assistenciais não-universitárias que respondam às demandas sociais por serviços, docência e tecnologia, especialmente em atividades mais ligadas aos níveis primário e secundário de atenção.

Outra necessidade é a mudança do estilo gerencial destas instituições. Os HU devem ser administrados tendo em vista sua integração com redes de provisão de cuidados primários e secundários, através:

a) da formalização e da subordinação aos sistemas de referência e contra-referência existentes, sejam eles definidos pelo planejamento das redes públicas de saúde, sejam organizados em função das necessidades de mercado pelas instituições mantenedoras de serviços de saúde;

b) da reorganização de suas funções de forma integrada com as necessidades de saúde de cada região;

c) da introdução de mecanismos que mudem as práticas gerenciais e o comportamento dos médicos e dos profissionais de saúde que nele participam;

Em muitos países tais soluções já vêm sendo adotadas, seja através da integração dos HU com Health Manteinance Organizations (modelo americano); seja através de sua relação com os sistemas de referência e contra-referência administrados por instituições de atenção primaria ou médicos de família, como é o caso dos GP's fund holders (modelo inglês).

Por fim, a integração dos HU com redes de atenção primária e secundária 
poderia ainda ajudar a redefinir e preservar o principal valor agregado que os hospitais universitários ainda podem trazer para os sistemas de saúde: o de serem centros de referência e alta tecnologia, contribuindo para o progresso técnico em saúde, especialmente nos países em desenvolvimento, onde as empresas e instituições científicas são débeis e desfinanciadas. Reduzir o papel dos HU a meros competidores da rede de saúde existente, duplicando de forma descoordenada e não-integrada todas às funções existentes nos sistemas de saúde, é um mau uso dos recursos que estão sendo destinados a estas instituições.

\section{SUMMARY}

\section{UNIVERSITY HOSPITALS: PAST, PRESENT} AND FUTURE

Purpose. This article discusses the evolution of university hospitals, their characteristics, functioning and financing and their role in medical education and research.

Methods. Data come from a workshop organized by the World Health Organization on university hospitals in 22 countries.

RESULTS. The main finding is that these institutions are increasingly inadequate to the reality of the health sector in which they operate and to the challenges posed by the profound changes currently taking place in the health system of most countries.

Conclusion. A reform is needed, and the article presents a few possible strategies for reforming these hospitals. [Rev Ass Med Brasil 200 |; 47(2): 149-56]

KEY WORDS: University hospitals. Medical Education.

\section{REFERÊNCIAS}

I. Institute for Health Policiy Studies - IEPSMorld Health Organization. The Proper Function of Teaching Hospitals Within Health Systems September, 1995.

2. Médici, A.C. A Economia da Demanda em Saúde. In A Economia Política das Reformas em Saúde. Cap. I, Ed. IAHCS, Porto Alegre (RS), 1997.

\section{IMAGEM EM MEDICINA}

Participe da nova seção da Ramb. É um espaço aberto ao leitor, que poderá enviar material de interesse educativo como fotos, ilustrações e exames, acrescido de três linhas explicativas contendo ainda nome do autor e serviço onde foi realizado. 0 material poderá ser enviado para a

\section{Rua São Carlos do Pinhal, 324 - Cep 01333-903 S. Paulo - SP - E-mail: ramb@amb.org.br}

